\title{
TAGUNG
}

\section{Zwischen Mitgliedstaaten und Völkerrecht: Die Umweltpolitik der Europäischen Gemeinschaft}

\author{
Joachim Bielitz*
}

Fragt man nach den Bereichen der Internationalen Politik und des Völkerrechts, in denen die Europäische Gemeinschaft eine hervorgehobene Rolle spielt, so ist neben der Liberalisierung des Welthandels und der Entwicklungshilfe die Umweltpolitik sicherlich an vorderster Stelle zu nennen. Es kann daher nicht verwundern, dass der AEI, der dem Tätigwerden von Union und Gemeinschaft auf internationaler Ebene derzeit in einer Tagungsreihe besondere Aufmerksamkeit zuteil werden lässt, dem Bereich der Umweltpolitik eine eigene Tagung widmete.

In seiner Einführung in die Thematik rief $P e$ ter-Christian Müller-Graff zunächst den Teilnehmern die Gründe für die Aktivitäten der Europäischen Gemeinschaft auf dem Gebiet der Umweltpolitik ins Gedächtnis: Umweltschutz könne allein auf nationaler Ebene nicht effektiv geleistet werden, sondern erfordere eine effiziente Kooperation auf supra- und internationaler Ebene. Als in rechtlicher Hinsicht besonders weit entwickelter Integrationsverbund sei daher die Gemeinschaft für die Wahrnehmung von Umweltschutzaufgaben in besonderer Weise geeignet. Zudem dürfe nicht übersehen werden, dass Umweltschutzmaßnahmen regelmäßig zu Wettbewerbsbeschränkungen führen und ein Tätigwerden der Gemeinschaft daher auch zum Schutz des innereuropäischen Binnenmarktes vor Wettbewerbsverzerrungen erforderlich sei. Müller-Graff erinnerte daran, dass dementsprechend die Gemeinschaft umweltbezogene Rechtsakte zunächst auf ihre Kompetenz zur Harmonisierung des Binnenmarktes ge-

\section{Die Europäische Gemeinschaft in der internationalen Umweltpolitik}

Interdisziplinäre Wissenschaftliche Tagung des Arbeitskreises Europäische Integration in Zusammenarbeit mit der Universität Würzburg Würzburg, 1.-2. Juli 2005

Wissenschaftliche Leitung

Prof. Dr. Dr. h.c. Peter-Christian MÜLLERGRAFF, AEI, Universität Heidelberg

Prof. Dr. Eckhard PACHE, Universität Würzburg Prof. Dr. Dieter H. SCHEUING, Universität Würzburg

\section{Grußwort}

Prof. Dr. Klaus LAUBENTHAL, Juristischen Fakultät, Universität Würzburg

Einführung in die Thematik

Prof. Dr. Dr. h.c. Peter-Christian MÜLLERGRAFF

Panel I: Grundlagen der Umweltpolitik der Europäischen Gemeinschaft

EG-Umweltpolitik im geltenden Primärrecht und im neuen Verfassungsvertrag

Prof. Dr. Astrid EPINEY, Universität Freiburg im Uechtland

Postulate und Konzepte: Zur Durchsetzbarkeit und Durchsetzung der EG-Umweltpolitik

Prof. Dr. Meinhard SCHRÖDER, Universität Trier

Die institutionellen Akteure der EG-Umweltpolitik auf der Grundlage der Binnenmarktintegration, des genuinen Umweltschutzes und der internationalen Umweltkooperation

Prof. Dr. Gisela MÜLLER-BRANDECKBOCQUET, Universität Würzburg

Die ökonomische Dimension der EG-Umweltpolitik

Dr. Hubert MANDERY, BASF AG, Ludwigshafen

* Joachim Bielitz, Wissenschaftlicher Mitarbeiter, Lehrstuhl für Staatsrecht, Völkerrecht, Internationales Wirtschaftsrecht und Wirtschaftsverwaltungsrecht, Universität Würzburg. 
stützt habe, bevor 1986 mit der Einheitlichen Europäischen Akte (EEA) ausdrückliche Kompetenzen der Gemeinschaft für den Bereich des Umweltrechts begründet wurden.

\section{Kohärenz und Effizienz als Leitmotive des Eu- ropäischen Umweltrechts}

Im Anschluss an diese Einführung war der erste Teil der Tagung den Grundlagen der Umweltpolitik der Europäischen Gemeinschaft gewidmet. Astrid Epiney gab einen Überblick über die Grundlagen der europäischen Umweltpolitik im geltenden Primärrecht und konzentrierte sich dabei insbesondere auf die Kompetenzen der Gemeinschaft bei der Binnenrechtsetzung. Als Rechtsgrundlagen kommen für die Rechtsetzung auf dem Gebiet des Umweltrechts insbesondere die Artt. 95 Abs. I und 175 Abs. II EGV in Betracht; doch ergibt sich eine Pflicht zur Berücksichtigung umweltpolitischer Belange auch bei der Rechtsetzung in anderen Politikbereichen aus der Querschnittsklausel des Art. 6 EGV. Epiney betonte die Notwendigkeit einer Abgrenzung zwischen den verschiedenen im konkreten Einzelfall in Betracht kommenden Zuständigkeiten sowohl auf Grund der unterschiedlichen Verfahrensanforderungen als auch wegen der divergierenden Möglichkeiten der Mitgliedstaaten zur Abweichung von Gemeinschaftsrecht nach Art. 95 einerseits und auf Grund der Schutzverstärkungsklausel nach Art. 176 andererseits. Maßgebliches Abgrenzungskriterium ist nach der Rechtsprechung des EuGH der Schwerpunkt der jeweiligen Regelung; der Weg der Doppelabstützung kann nur dann gewählt werden, wenn ein Schwerpunkt nicht zu ermitteln ist und hinsichtlich des Verfahrens und der Schutzverstärkungsoptionen keine Divergenzen zwischen beiden Rechtsgrundlagen bestehen.

Im Folgenden veranschaulichte Epiney die Kompetenzproblematik an zwei aktuellen und - auch in der folgenden Diskussion - durch-
Panel II: Europäische Gemeinschaft und internationale Umweltpolitik

Spektrum und Status der internationalen Umweltkonventionen

Prof. Dr. Wolff HEINTSCHEL VON HEINEGG, Europa-Universität Viadrina, Frankfurt/Oder

Die Kompetenzen der EG für internationale Umweltpolitik

Siegfried BREIER, Europäische Kommission, Brüssel

Handlungsspielräume und Verpflichtungskonflikte im Spannungsfeld zwischen internationalen Abkommen und Gemeinschaftsrecht: das Beispiel der Kerosinsteuerproblematik

Prof. Dr. Eckhard PACHE, Universität Würzburg

Umsetzungsprobleme internationaler Umweltschutzkonventionen: das Beispiel des Kyoto-Protokolls - Emissionshandel als Instrument internationaler, europäischer und staatlicher Umweltpolitik

Dr. Wolfgang SEIDEL, Bundesumweltamt, Berlin

Kann die Europäische Union ihre Vorreiterrolle in der internationalen Klimapolitik glaubhaft fortsetzen?

Dr. Axel MICHAELOWA, Hamburgisches Welt-Wirtschafts-Archiv

aus kontrovers beurteilten Beispielen: Nicht als eine Frage der Abgrenzung verschiedener Zuständigkeiten, sondern als eine Frage der Reichweite der Rechtsetzungskompetenz aus Art. 175 Abs. II EGV erscheint die Problematik, ob der Gemeinschaft eine Kompetenz zur Einführung einer altruistischen Verbandsklage im Bereich des Umweltrechts zukommt. Während gegen eine solche Zuständigkeit vielfach der Grundsatz der Verfahrensautonomie der Mitgliedstaaten angeführt wird, vertrat Epiney die Auffassung, dass diesem Grundsatz keine rechtlich eigenständige $\mathrm{Be}-$ deutung zukomme. Die Zulässigkeit derartiger Verfahrensregelungen ergebe sich bereits aus Art. 175 Abs. IV EGV; im Übrigen trenne das Gemeinschaftsrecht nicht in einer dem deutschen Recht vergleichbaren Weise zwischen materiellem Recht und dessen Durchsetzung. Da die Einführung einer Verbands- 
klage grundsätzlich der Verwirklichung der Ziele des Art. 174 diene, sei eine Zuständigkeit gemäß Art. $175 \mathrm{zu}$ bejahen; freilich begrenzt auf eine Verbandsklage im Bereich des gemeinschaftsrechtlich determinierten Umweltrechts.

Als zweites Beispiel wählte Epiney die Problematik der Einführung von Nutzungsentgelten, also von Entgelten für die Verursachung von Beeinträchtigungen globaler Umweltgüter. Für die Frage nach der für die Einführung derartiger Nutzungsentgelte einschlägigen Rechtsgrundlage kommt es nach Epineys Auffassung maßgeblich auf den Anknüpfungspunkt derartiger Entgelte an. So sei bei produktbezogenen Entgelten, die sich zum Beispiel am Treibstoffverbrauch orientieren, Art. 93 EGV (Einstimmigkeitserfordernis) anzuwenden, während bei in Anknüpfung an das Emissionsverhalten oder die Infrastrukturnutzung erhobenen Nutzungsentgelten auf Grund des dominierenden Umweltbezuges Art. 175 EGV vorzugswürdig sei. Innerhalb des Art. 175 EGV wiederum sei wegen des Gegenleistungsbezuges derartiger Abgaben die Steuerklausel des Absatzes II nicht einschlägig, Kompetenzgrundlage sei vielmehr Absatz I (Mitentscheidungsverfahren). Die Sinnhaftigkeit einer unterschiedlichen Bewertung von Emissionsabgaben einerseits und Abgaben auf den Treibstoffverbrauch wurde freilich in der folgenden Diskussion wegen der sachlichen Nähe beider Anknüpfungspunkte kritisch in Frage gestellt.

\section{EG-Umweltrecht: Rechtsdurchsetzung, von unten"}

Im Mittelpunkt des nachfolgenden Vortrags von Meinhard Schröder standen die verschiedenen Instrumente und rechtspolitischen Strategien zur Sicherstellung der effektiven Durchsetzung des Gemeinschaftsrechts in den Mitgliedstaaten. Als eines der insoweit wichtigsten Konzepte nannte Schröder die Mobilisierung des Einzelnen als Instrument der Vollzugskontrolle; ein Konzept, das in der Direktwirkung von Richtlinien ebenso zum Ausdruck käme wie in der Staatshaftung für
Verstöße gegen Gemeinschaftsrecht. Insbesondere mit Blick auf die Direktwirkung von Richtlinien sei wegen der insoweit sehr großzügigen Rechtsprechung des EuGH davon auszugehen, dass dieses Rechtsinstitut primär im Kontext der ,Mobilisierungsstrategie " und weniger als Instrument des effektiven Individualrechtsschutzes zu sehen sei. Auch durch die Zuerkennung verfahrensunabhängiger Informationsansprüche (zum Beispiel durch die Umweltinformations-Richtlinie) wie auch verfahrensabhängiger Beteiligungsrechte (zum Beispiel durch die UVP- und Plan-UVPRichtlinie) könne die Öffentlichkeit als Kontrollinstanz nutzbar gemacht werden. Schröder schloss sich der Auffassung von Epiney an, dass auch die Einführung einer Verbandsklage ein sinnvolles Instrument zur Durchsetzung des Umweltrechts sein könne, und verwies auch auf die Garantien der sowohl von der Gemeinschaft als auch von den Mitgliedstaaten unterzeichneten Aarhus-Konvention.

Einen Perspektivwechsel ermöglichten die folgenden Referate von Gisela Müller-Brandeck-Bocquet und Hubert Mandery, die die Umweltpolitik der Gemeinschaft aus politologischer Sicht beziehungsweise aus der Sicht der Wirtschaft beleuchteten. Dabei machte das Referat von Müller-Brandeck-Bouquet deutlich, dass die Abgrenzung verschiedener Kompetenzgrundlagen und die daraus resultierenden Verfahrensunterschiede nicht nur aus juristischem, sondern auch aus politologischem Blickwinkel von eminenter Bedeutung sind. Insbesondere das Europäische Parlament habe sich während der vergangenen zwei Jahrzehnte als ,umweltpolitisches Gewissen“ der Gemeinschaft erwiesen, so dass die Ausweitung des Mitentscheidungsverfahrens tendenziell dem Umweltschutz zugute komme. Mandery wiederum rief den Teilnehmern der Tagung nachdrücklich ins Gedächtnis, dass man bei der Beurteilung der Sinnhaftigkeit umweltpolitischer und -regulatorischer Maßnahmen stets auch die durch derartige Maßnahmen verursachten Kosten in Relation zum mutmaßlichen ökologischen Nutzen im Auge behalten müsse. 


\section{Entdynamisierung des Umweltvölkerrechts}

Auf der durch diese vier Vorträge gelegten Grundlage konnten im zweiten Teil der Tagung verschiedene Aspekte der Mitwirkung der Gemeinschaft in der internationalen Umweltpolitik vertieft werden. Einführend gab Wolff Heintschel von Heinegg einen Überblick über die Entwicklung des Umweltvölkerrechts und die unter Beteiligung der Gemeinschaft abgeschlossenen umweltvölkerrechtlichen Verträge. Als zentralen Auslöser für die in der Entwicklung des Umweltvölkerrechts liegende Zurücknahme der nationalen Souveränität hob von Heinegg die Tatsache hervor, dass eine gemeinsame Nutzung globaler Umweltressourcen zwingend sei - Umweltprobleme respektierten bekanntermaßen keine politischen Grenzen. Insbesondere im Anschluss an die Stockholmer Konferenz über die Umwelt des Menschen von 1972 wurde eine Vielzahl universeller völkerrechtlicher Verträge abgeschlossen. Stand dabei zunächst die Umwelt als Schutzgut um ihrer selbst willen im Mittelpunkt, so kam es mit der auch als ,Erdgipfel ' bezeichneten Konferenz von Rio de Janeiro 1992 zu einer verstärkten Verknüpfung von Umweltschutz und Entwicklungshilfe, die zu einer Privilegierung der Entwicklungsländer in zahlreichen umweltvölkerrechtlichen Verträgen führte. Zugleich traten in den Neunzigerjahren Spannungen zwischen wirtschafts- und umweltvölkerrechtlichen Regelungen deutlicher als zuvor zutage. Insgesamt sei derzeit eine Verlangsamung in der Entwicklung des Umweltvölkerrechts zu beobachten, die gleichermaßen auf den bereits erreichten hohen Kodifikationsstand wie auch auf wirtschaftsund entwicklungspolitische Einflüsse zurückzuführen sei. Eine Fortentwicklung des Umweltvölkerrechts sei daher auf absehbare Zeit vorrangig auf regionaler Ebene zu erwarten. Ganz überwiegend nur von regionaler Bedeutung sei schließlich auch das umweltbezogene Völkergewohnheitsrecht; die Deklarierung zum Beispiel des Vorsorgeprinzips als ein weltweit gültiger Satz des Völkergewohn- heitsrechts sei politisches Wunschdenken fernab der rechtlichen Realität.

\section{Umweltvölkerrecht im europäischen Kontext}

Im folgenden Referat beschäftigte sich Siegfried Breier von der Europäischen Kommission ausführlich mit den Kompetenzen der Gemeinschaft für die Internationale Umweltpolitik. Breier ging zunächst auf den eng begrenzten Anwendungsbereich des Art. 174 Abs. IV EGV als einziger expliziter Außenkompetenz der Gemeinschaft im Umweltbereich ein, um sich danach den impliziten Kompetenzen der Gemeinschaft zuzuwenden. Derartige implizite Außenkompetenzen ergeben sich nach der AETR-Rechtsprechung des EuGH insbesondere aus der Parallelität von Innen- und Außenkompetenzen, wenn die Gemeinschaft von ihren Zuständigkeiten für die Binnenrechtsetzung Gebrauch gemacht hat. Die Abhängigkeit der Anerkennung einer Außenkompetenz von einer entsprechenden Rechtsetzungstätigkeit der Gemeinschaft hat allerdings zur Folge, dass die allermeisten umweltvölkerrechtlichen Abkommen als gemischte Abkommen unter Beteiligung der Gemeinschaft und der Mitgliedstaaten geschlossen werden, da der Inhalt von Gemeinschaftsrechtsakten und völkerrechtlichen Abkommen nur selten deckungsgleich ist und eine ausschließliche Kompetenz der Gemeinschaft aus diesem Grund regelmäßig nicht besteht.

Die EG als Umweltvölkerrechtssubjekt: zunehmende Verpflichtungskonflikte

Hatten bis dahin die Kompetenzen der Gemeinschaft im Mittelpunkt gestanden, so beschäftigte sich Eckhard Pache im nachfolgenden Vortrag mit den völkerrechtlichen Bindungen der Gemeinschaft. Am Beispiel der auch in jüngster Zeit auf politischer Ebene wieder diskutierten Einführung einer Kerosinbesteuerung zeigte Pache auf, dass erstens völkerrechtliche Vorgaben für Rechtsetzungsakte der Gemeinschaft mit Umweltbezug sich nicht nur aus dem Umweltvölkerrecht, sondern ebenso auch aus dem Wirtschaftsvölkerrecht 
und dem Internationalen Luftrecht ergeben können und dass zweitens die Gemeinschaft insoweit nicht nur an die von ihr selbst, sondern auch an die von ihren Mitgliedstaaten geschlossenen völkerrechtlichen Verträge gebunden sein kann. Derartige Bindungen der Gemeinschaft an völkerrechtliche Verträge ihrer Mitgliedstaaten können sich nicht nur aus einem formellen Beitritt der Gemeinschaft zu den jeweiligen Verträgen ergeben (der häufig bereits deshalb nicht in Betracht kommt, weil viele Abkommen nur Staaten zum Beitritt offen stehen), sondern auch aus einer informellen (Teil-) Rechtsnachfolge der Gemeinschaft in Verträge ihrer Mitgliedstaaten sowie schließlich auch aus gemeinschaftsrechtlichen Rücksichtnahmepflichten.

Eine informelle (Teil-) Rechtsnachfolge kommt freilich weder für Inter-se-Verträge der Mitgliedstaaten in Betracht (insoweit gilt vielmehr der Vorrang des Gemeinschaftsrechts) noch für bilaterale Verträge der Mitgliedstaaten mit Drittstaaten, da eine Rechtsnachfolge in von verschiedenen Mitgliedstaaten mit einem Drittstaat abgeschlossene Verträge im Detail unterschiedlichen Inhaltes zu schwerwiegenden Problemen führen würde. Eine informelle (Teil-) Rechtsnachfolge in mulilaterale, unter Beteiligung aller Mitgliedstaaten abgeschlossene Verträge erscheint hingegen grundsätzlich möglich; zur Bestimmung der diesbezüglichen Voraussetzungen kann auf die Rechtsprechung des EuGH zur Stellung der Gemeinschaft im GATT 1947 sowie auf die im Völkerrecht geltenden Grundsätze zur Staatennachfolge zurückgegriffen werden.

Gemeinschaftsrechtliche Pflichten zur Beachtung der von den Mitgliedstaaten eingegangenen völkerrechtlichen Bindungen können sich für kompetenzgemäß abgeschlossene Altverträge zunächst aus Art. 307 EGV ergeben. Pache betonte die in dieser Norm grundsätzlich angelegte zeitliche Begrenzung der Rücksichtnahmepflicht durch die Pflicht der Mitgliedstaaten zur Anpassung ihrer Verträge, wies jedoch zugleich darauf hin, dass die Er- füllung der Anpassungspflicht bei multilateralen Verträgen häufig praktisch unmöglich sei. Schlössen hingegen die Mitgliedstaaten völkerrechtliche Verträge unter Missachtung der Kompetenzen der Gemeinschaft, so greife Art. 307 nicht ein; eingeschränkte Rücksichtnahmepflichten wie auch verstärkte Anpassungspflichten würden sich in diesem Fall aus Art. 10 EGV ergeben.

\section{,Effizienzgrenzen` - hohe Kontrollkosten bei der Umweltvölkerrechtsdurchsetzung}

Das Zusammenspiel von Völkerrecht, Gemeinschaftsrecht und nationalem Recht stand auch im Mittelpunkt des Vortrags von Wolfgang Seidel von der Deutschen Emissionshandelsstelle im Umweltbundesamt, der die sich daraus ergebenden Probleme am Beispiel des in der Gemeinschaft 2005 angelaufenen Emissionshandels schilderte. Zu Beginn seines Vortrages ging Seidel zunächst auf die umweltökonomischen Grundlagen des Emissionshandels ein: Grundgedanke des Emissionshandels ist die Verwirklichung von Umweltschutzzielen durch Marktmechanismen, die eine möglichst kosteneffiziente Reduzierung des $\mathrm{CO}_{2}$-Ausstoßes ermöglichen soll, und zugleich die Internalisierung der durch Umweltschutzmaßnahmen verursachten Kosten. Vor diesem Hintergrund stellt der Emissionshandel ein Bewirtschaftungssystem ein, das sich durch die Kernelemente der mengenmäßigen Begrenzung der handelbaren Emissionszertifikate (,cap') und die Möglichkeit des freien Handels mit derartigen Zertifikaten (,trade') auszeichnet. Als Hauptprobleme des Emissionshandels aus regulatorischer Sicht nannte Seidel die Bestimmung der Gesamtemissionsmenge, also des ,cap', die Kriterien für die Zuteilung der Zertifikate und die Schaffung geeigneter Mechanismen zur Überwachung und Kontrolle des Handelssystems. Während der weltweite Emissionshandel nach Maßgabe des Kyoto-Protokolls erst im Jahr 2008 anlaufen soll, hat der Emissionshandel auf europäischer Ebene bereits begonnen. Seidel gab einen Überblick über den sachlichen und sektoralen Anwendungsbereich der Euro- 
päischen Emissionshandels-Richtlinie und ging nachfolgend insbesondere auf die Kriterien für die Zuteilung der Berechtigungen ein, die in der Gemeinschaft für die erste Verpflichtungsperiode von 2005 bis 2007 aus Gründen des Bestandsschutzes weitestgehend unentgeltlich auf der Basis historischer Emissionen und nicht durch eine Versteigerung von Zertifikaten erfolgte. Abschließend stellte Seidel die zahlreichen Schwierigkeiten dar, die sich in der Verwaltungspraxis bei der Zuteilung der Emissionszertifikate in Deutschland auf der Basis des TreibhausEmissionshandelsgesetzes ergeben hätten.

Nachlassendes EG-Engagement fürs KyotoProtokoll

Zum Abschluss der Tagung referierte Axel Michaelowa über die derzeitigen Bemühungen der Gemeinschaft, eine einheitliche Position für die Verhandlungen über die Folgevereinbarungen zum Kyoto-Protokoll zu entwickeln, und bot damit einen Ausblick auf die Internationale Klimapolitik der kommenden Jahre. Fazit seines Beitrages war, dass die Gemeinschaft zwar für sich beanspruchen könne, insbesondere bei der Aushandlung des Kyoto-Protokolls eine Vorreiterrolle gespielt zu haben, gegenwärtig aber im Begriff sei, diese Vorreiterrolle durch unzureichende Zielsetzungen für die Verpflichtungsperiode ab 2008 zu verspielen.

\section{Erhöhte Regelungsdichte bleibt problematisch}

Fasst man die Ergebnisse dieser Tagung zusammen, so bleibt festzuhalten, dass das Umweltrecht wie nur wenige andere Rechtsgebiete durch das Zusammenwirken von Völkerrecht, Gemeinschaftsrecht und nationalem Recht geprägt ist. Daraus wie auch aus dem Einsatz neuartiger Regulierungsinstrumente zum Beispiel im Emissionshandel ergibt sich eine Vielzahl rechtlicher Probleme, die das Umweltrecht als ein aus rechtswissenschaftlicher Perspektive besonders spannendes Rechtsgebiet erscheinen lässt, zugleich aber auch eine Erklärung für die aus umweltpolitischer Sicht bisweilen nur unbefriedigenden Fortschritte unter anderem im Bereich des Klimaschutzes bietet.

Die einzelnen Beiträge der Tagung werden in Kürze in ausführlicher Form in einem Band der Schriftenreihe des AEI beim Nomos Verlag veröffentlicht. 